\title{
Sonic hedgehog-dependent activation of Gli2 is essential for embryonic hair follicle development
}

\author{
Pleasantine Mill, ${ }^{1}$ Rong Mo, ${ }^{1}$ Hong Fu, ${ }^{1}$ Marina Grachtchouk, ${ }^{2}$ Peter C.W. Kim, ${ }^{3}$ \\ Andrzej A. Dlugosz, ${ }^{2}$ and Chi-chung Hui ${ }^{1,4}$ \\ ${ }^{1}$ Program in Developmental Biology, The Hospital for Sick Children, and Department of Molecular and Medical Genetics, \\ University of Toronto, Toronto, Ontario M5G 1X8, Canada; ${ }^{2}$ University of Michigan Comprehensive Cancer Center and \\ Department of Dermatology, Ann Arbor, Michigan 48109, USA; ${ }^{3}$ Program in Infection, Immunity and Repair, The Hospital \\ for Sick Children, and Department of Surgery, University of Toronto, Toronto, Ontario M5G 1X8, Canada
}

\begin{abstract}
Sonic hedgehog (Shh) signaling plays a critical role in hair follicle development and skin cancer, but how it controls these processes remains unclear. Of the three Gli transcription factors involved in transducing Shh signals in vertebrates, we demonstrate here that Gli2 is the key mediator of Shh responses in skin. Similar to $S_{h h}^{-/-}$mice, Gli2-/- mutants exhibit an arrest in hair follicle development with reduced cell proliferation and Shh-responsive gene expression, but grossly normal epidermal differentiation. By transgenic rescue experiments, we show that epidermal Gli2 function alone is sufficient to restore hair follicle development in $\mathrm{Gli2}^{-/-}$skin. Furthermore, only a constitutively active form of Gli2, but not wild-type Gli2, can activate Shh-responsive gene expression and promote cell proliferation in $S_{h h^{-/}}$skin. These observations indicate that Shh-dependent Gli2 activator function in the epidermis is essential for hair follicle development. Our data also reveal that Gli2 mediates the mitogenic effects of Shh by transcriptional activation of $c y c l i n D 1$ and cyclin $D 2$ in the developing hair follicles. Together, our results suggest that Shh-dependent Gli2 activation plays a critical role in epithelial homeostasis by promoting proliferation through the transcriptional control of cell cycle regulators.
\end{abstract}

[Keywords: Shh; Gli2; hair follicle; mitogen; D-type cyclins]

Supplemental material is available at http://www.genesdev.org.

Received September 4, 2002; revised version accepted November 14, 2002.

The development of hair follicles during embryogenesis is dependent on reciprocal inductive interactions between the epithelium and mesenchyme (Hardy 1992). Classical studies propose that a signal originating from the mesenchyme instructs specific clusters of ectodermal cells to elongate into epithelial placodes. The placodes then signal to the underlying mesenchyme directing the condensation of fibroblasts into rudimentary dermal papillae, and subsequently, the dermal condensates stimulate the proliferation and differentiation of epithelial cells. In postnatal skin, epithelium of the hair follicle continuously cycles through periods of active growth (anagen), followed by regression (catagen) and a resting phase (telogen). At the beginning of each anagen, stem cells located in the bulge of the hair follicles respond to signals from the dermal papillae and give rise to both follicular and epidermal keratinocytes (for review, see

${ }^{4}$ Corresponding author.

E-MAIL: cchui@sickkids.ca; FAX (416) 597-9497.

Article and publication are at http://www.genesdev.org/cgi/doi/10.1101/ gad.1038103.
Fuchs and Raghavan 2002). Recent experiments indicate that multiple epithelial and mesenchymal signals are involved in these inductive interactions (for review, see Millar 2002). Many of the signaling cascades involved in embryonic hair development are redeployed during adult hair development, and hyperactivation or inappropriate maintenance of these signaling pathways can result in skin disorders and cancers.

The secreted signaling molecule Sonic hedgehog (Shh) plays an important role in both embryonic and adult hair development. During embryogenesis, Shh is expressed in the proliferating epithelial cells at the distal tip of the developing follicle (Bitgood and McMahon 1995; Iseki et al. 1996). In mice lacking Shh, mature hair follicles fail to develop although hair follicle formation is initiated and the dermal condensates form, suggesting that Shh is required for the proliferation and subsequent downgrowth of the follicular epithelium, and for the maturation of the dermal papillae (St-Jacques et al. 1998; Chiang et al. 1999; Karlsson et al. 1999). In adult mice, Shh expression is up-regulated in early anagen, and ectopic application of Shh can prematurely induce anagen in rest- 
ing telogen follicles (Sato et al. 1999). Furthermore, treatment with Shh-blocking antibodies causes a reversible alopecia, as follicles cannot cycle and are arrested in telogen, indicating that Shh is also required for hair cycling in postnatal skin (Wang et al. 2000b). Importantly, hyperactivation of the Shh signaling pathway is found in a variety of hair follicle tumors, including basal cell carcinomas (BCCs), the most common human cancers (Dahmane et al. 1997; Oro et al. 1997; Xie et al. 1998).

The Gli family of zinc finger transcription factors mediates Hedgehog (Hh) signaling in Drosophila and vertebrates (Aza-Blanc and Kornberg 1999; Ingham and McMahon 2001). In Drosophila, a single Gli protein Cubitus interruptus (Ci) possesses both activator and repressor functions. The repressor form is generated by proteolytic cleavage of $\mathrm{Ci}$, which is inhibited by Hh signaling (AzaBlanc et al. 1997). In addition to blocking the formation of the Ci repressor, Hh signaling also promotes the activator function of Ci through poorly understood molecular events (see Ingham and McMahon 2001). In mammals, Shh signal transduction involves at least three Gli proteins (Gli1, Gli2, and Gli3). Similar to Ci, Gli2 and Gli3 likely function as both activators and repressors. In contrast, Glil appears to be solely an activator. It is believed that Gli2 and Gli3 are the primary transducers of Shh signaling, whereas Gli1, whose expression is transcriptionally regulated by Gli2 and Gli3, plays a secondary role in potentiating the Shh response. Mutant studies revealed that Gli2 and Gli3 perform most of the activator and repressor functions, respectively, in developing mouse embryos (Ding et al. 1998; Motoyama et al. 1998a; Bai and Joyner 2001). In contrast, Gli1 function is dispensable for development, as Gli1 null mice are viable and do not exhibit any anomalies (Park et al. 2000). However, the three Gli proteins possess overlapping developmental functions, as both Gli1;Gli2 and Gli2;Gli3 mutant mice exhibit more severe phenotypes than the single mutant mice (Mo et al. 1997; Motoyama et al. 1998a; Park et al. 2000).

Although Shh signaling plays a critical role in both embryonic and adult hair development, the function of Gli transcription factors in these processes is not known. Molecular analysis of BCC has revealed that overexpression of Gli1 and, to a lesser extent, Gli2 are common features of BCC and indicators of hyperactivated Shh signaling (Dahmane et al. 1997; Grachtchouk et al. 2000). Indeed, overexpression of Gli1 and Gli2 in the mouse epithelium resulted in the formation of BCC-like tumors, indicating that the activator functions of Gli transcription factors play a key role in the initiation of these basaloid tumors in postnatal skin (Grachtchouk et al. 2000; Nilsson et al. 2000). To determine the role of Gli proteins in embryonic hair development, we have analyzed the phenotypes of Gli2, Gli3, and Shh mutant skin, and performed transgenic rescue experiments. We show that Gli2 is the key mediator of Shh signaling during embryonic hair development as $\mathrm{Gli2}^{-/-}$skin phenocopies the arrested hair follicle phenotype of $S_{h}{ }^{-/-}$mutants, with reduced Shh target gene expression and cell proliferation. Our results also demonstrate that the hair fol- licle defects of Gli2-/- skin are primarily in the epithelium. Importantly, we find that the activation of Gli2 is Shh-dependent and is tightly linked to the control of cell proliferation, partly through transcriptional regulation of the cell cycle regulators cyclin D1 and cyclin D2.

\section{Results}

Gli2 function is essential for hair follicle development

To identify the Gli transcription factors that mediate Shh responses during hair follicle development, we examined the skin phenotypes of Gli2 and Gli3 mutant mice. Similar to wild-type skin, epithelial placodes form around embryonic day 14.5 (E14.5) in $\mathrm{Shh}^{-/-}, \mathrm{Gli2}^{-/-}$, and Gli3 $^{-/-}$skin (Fig. 1A-D). At E18.5, although both wildtype and $\mathrm{Gli3}^{-/-}$skin contains follicles at all stages of development (stages 1-5; Fig. 1E,H), all $S h^{-/-}$follicles and the majority of $\mathrm{Gli2}^{-/-}$follicles are arrested at the early hair plug stage (Fig. 1F,G). Advanced-stage wildtype and Gli3 $^{-1-}$ follicles grow deep into the dermis and show compartmental organization and maturation of the dermal papilla, as indicated by a high level of alkaline phosphatase activity (Fig. 1I,L). In contrast, only rudimentary mesenchymal condensates are found in $S h^{-/-}$ and Gli2 $2^{-/-}$follicles (Fig. 1J,K). However, some "escape" hairs, which develop to more advanced stages, can be found in E18.5 Gli2 $^{-/-}$skin; about $8 \%$ of Gli2 $^{-/-}$follicles develop to stage 4 or higher $(\mathrm{p}<0.005)$ as compared with $77.5 \%$ of wild-type follicles (Fig. 4B, below, and summarized in Fig. 4W). Similar to $\mathrm{Shh}^{-/-}$skin, $\mathrm{Gli2}^{-/-}$skin also shows a significant reduction $(25 \%$; $<<0.001)$ of pelage follicles as compared with wild-type littermates (data not shown).

Although pelage (trunk) follicle morphogenesis is completely abolished in $S h h^{-/-}$skin, we find that vibrissae (whisker) follicles can develop in $\mathrm{Shh}^{-1-}$ mice as well as in Gli2 $^{-/-}$mice. As revealed by $\mathrm{K} 17$ expression, which marks all developing epithelial appendages at E14.5, wild-type whisker pad on the upper lip consists of a vertical row of four large follicles alternating with five rows of varying numbers of follicles (Fig. 1M). Gli2 ${ }^{-/-}$mice have fewer and less developed vibrissae follicles as revealed by $K 17$ expression (Fig. 1O). Despite their profound craniofacial defect, $S h h^{-/-}$mice develop vibrissae follicles (Fig. 1N,S). In contrast, Gli3 ${ }^{-/-}$mice have extra vibrissae follicles (Fig. 1P). Therefore, although the developmental mechanisms of vibrissae and pelage follicles appear similar (Davidson and Hardy 1952), the requirement for Shh signaling is different.

Because $\mathrm{Gli2}^{-/-}$mice die embryonically, postnatal skin development was analyzed by xenografting experiments. This strategy allowed us to investigate whether or not (1) the arrest in folliculogenesis was due to a developmental delay, and (2) the follicle defect was secondary to a systemic defect in Gli2 ${ }^{-/-}$mice. Skin biopsies, consisting of both the epithelium and dermis, were removed from E18.5 embryos, grafted onto the back of immunocompromised mice, and allowed to further develop for 3-5 wk. Both wild-type $(\mathrm{n}=4)$ and $\mathrm{Gli3}^{-/-}(\mathrm{n}=5)$ 
Mill et al.

Figure 1. Arrest of Gli2 $^{-/-}$pelage follicle development phenocopies the skin defects of $\mathrm{Shh}^{-1-}$ mutants. Development of pelage follicles and whiskers in wild-type $(A, E, I$, $M, R), S^{-1-}(B, F, J, N, S), \quad G l i 2^{-/-}(C, G, K$, $O, T)$, and $G l i 3^{-/-}(D, H, L, P, U)$ mutants. $(A-$ $D)$ Pelage follicle development in E14.5 skin. Yellow line marks the boundary between epithelium (e) and dermis (d). $(E-H)$ Pelage follicle development in E18.5 skin. Numbers denote stage of hair follicle morphogenesis. $(I-L)$ E18.5 skin sections stained for alkaline phosphatase (blue) and laminin-5 (red). Arrowheads mark the developing dermal papilla, which show high alkaline phosphatase activity. $(M-P)$ Patterning of vibrissae follicles in E14.5 heads as revealed by $K 17$ RNA expression. In $M$, numbers indicate the normal tract pattern and arrowheads mark the four intervening whiskers. Arrow indicates the whisker formed in $\mathrm{Shh}^{-1-}$ mice $(N)$, and asterisk marks the extra whisker tract in Gli3 $^{-/-}$ mutants $(P)$. $(R-U)$ Histology of vibrissae follicles in E18.5 skin. Arrow indicates the characteristic concentric rings on crosssections of $\mathrm{Shh}^{-/-}$vibrissae follicles. Bars, $50 \mu \mathrm{m}$.

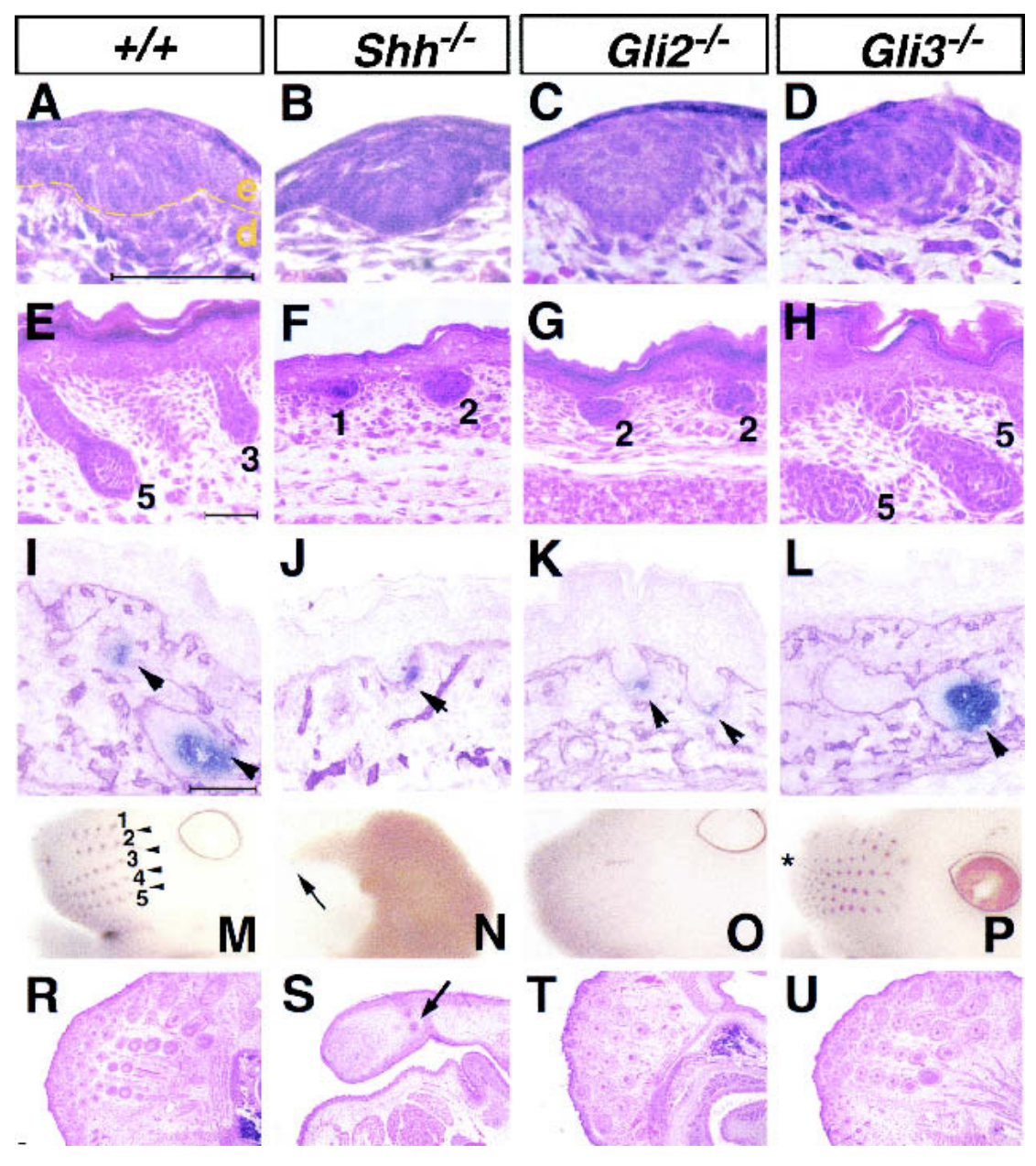

consistent reduction in cell proliferation throughout folliculogenesis, as shown by bromodeoxyuridine (BrdU) incorporation (Fig. 2A-D), and expression of proliferating cell nuclear antigen (PCNA; Fig. 2E,F) and Ki67 (data not shown). Although both epithelial and dermal layers in Gli2 $^{-/-}$skin show reduced proliferation, only the follicular epithelium was significantly affected (Fig. 2I). The expression of keratinocyte differentiation markers K5, $\mathrm{K} 10$, and loricrin, which mark the stratum basale, stratum spinosum, and stratum corneum, respectively, are not affected in $\mathrm{Gli2}^{-/-}$skin (Fig. 2A-H). These results indicate that keratinocyte differentiation proceeds normally in Gli2 $^{-/-}$skin. Furthermore, TUNEL staining revealed that the arrest in Gli2 ${ }^{-/-}$follicle development is not due to altered apoptosis (data not shown). These results show that Gli2 plays an essential role in cell proliferation, but not in keratinocyte differentiation and cell death, in the developing follicle.

\section{Gli2 mediates the majority of Shh responses in the developing skin}

To determine whether Gli2 is responsible for transducing Shh signals in embryonic skin, we examined expression of two universal Shh targets, Ptc1 and Gli1, in 


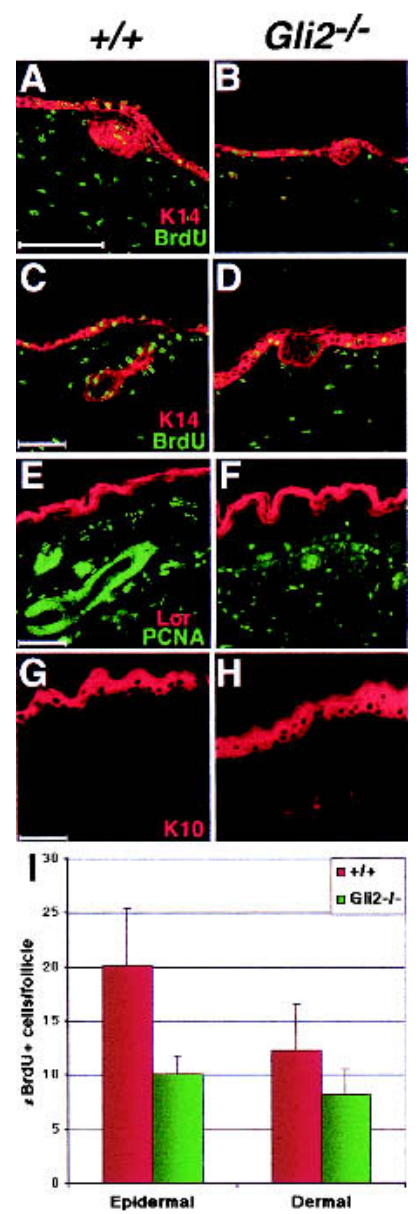

Figure 2. Gli2 ${ }^{-/-}$mutant follicles are arrested because of a defect in proliferation. Immunofluorescence of differentiation, and cell proliferation markers of wild-type $(A, C, E, G)$ and $G l i 2^{-/-}$ $(B, D, F)$ skin at E14.5 $(A-B)$ and E18.5 $(C-H) .(A-D)$ Staining of Keratin14 (red) and BrdU (green) at E14.5 $(A, B)$ and E18.5 $(C, D)$. $(E-F)$ Staining of Loricrin (red) and PCNA (green) in E18.5 skin. $(G-H)$ Staining of Keratin10 in E18.5 skin. (I) BrdU-positive cells in E18.5 skin. Gli2 ${ }^{-/-}$hair follicles show a twofold reduction in epithelial proliferation $(\mathrm{p}<0.0005)$. Bars, $50 \mu \mathrm{m}$.

Gli2 $^{-/-}$skin and in cultured dermal fibroblasts derived from Gli2 ${ }^{-/-}$mice. Ptc1 and all three Gli genes are highly expressed in both the epithelial and mesenchymal compartments of early hair follicles (Hui et al. 1994; data not shown). In later stage follicles, the expression levels of Ptc1 and Gli1 remain high in the developing epithelial compartments and dermal papilla, with lower levels lingering in the interfollicular layers (Fig. 3B,D). In contrast, the expression levels of Gli2 and Gli3 are dramatically reduced in both germ layers, with Gli2 being expressed at a slightly higher level in the developing follicle (Fig. $3 \mathrm{E}, \mathrm{F})$. Previous studies have shown that the skin expression of Ptc1 and Gli1 is almost completely lost in $\mathrm{Shh}^{-/-}$ mice (St.-Jacques et al. 1998; Chiang et al. 1999). Although Shh is still strongly expressed in $\mathrm{Gli}^{-/-}$follicles (Fig. 3G), expression of Ptc1 and Gli1 is greatly reduced in $\mathrm{Gli}^{-/-}$skin (Fig. $3 \mathrm{H}, \mathrm{J}$ ) suggesting that Gli2 mediates most of the Shh responses during folliculogenesis. In contrast, a low level of Gli3 expression, which is comparable to that detected in wild-type follicles, can be detected in Gli2-/- follicles (Fig. 3F,L). Similar to $\mathrm{Shh}^{-/-}$ mutants (St.-Jacques et al. 1998), the expression of Ptc2 is also not affected in Gli2 ${ }^{-/-}$follicles (Fig. 3I).

To further establish the role of Gli2 in mediating Shh responses in the skin, we isolated dermal fibroblasts from E18.5 wild-type and $\mathrm{Gli}^{-/-}$skin, and we examined the Shh-dependent activation of Gli1 and Ptc1 expression in primary cultures. When treated with recombinant Shh, wild-type fibroblasts exhibited a dose-dependent increase of Gli1 and Ptc1 expression, as revealed by reverse transcriptase PCR (RT-PCR) analysis (Fig. 3M). In contrast, Gli2 $2^{-/}$fibroblasts failed to show any increase of Gli1 and Ptc1 expression even at the highest Shh concentration tested. These results indicate that Gli2 is critical for transducing Shh signals in both epithelial and mesenchymal cells in skin.

\section{Epidermal Gli2 rescues the follicular defects of $\mathrm{Gli}^{-/-}$skin}

Shh signaling plays a key role in the proliferation of epithelial cells: Shh promotes keratinocyte proliferation by opposing cell cycle arrest in culture (Fan and Khavari 1999|; abnormal activation of Shh signaling results in the formation of basaloid tumors of epithelial origin in both humans and mice (Dahmane et al. 1997; Oro et al. 1997; Xie et al. 1998; Grachtchouk et al. 2000; Nilsson et al., 2000); and both $\mathrm{Shh}^{-/-}$and $\mathrm{Gli}^{-/-}$mice have profound proliferation defects in the epithelial compartment of the follicles (St-Jacques et al. 1998; Fig. 2A-F). To further define the function of Gli2 in the developing skin, we examined whether the K5-Gli2 transgene, which targets Gli2 expression to the epithelial basal layer and outer root sheath of differentiated hair follicles (Grachtchouk et al. 2000), can restore Shh responsiveness in the epithelium of $\mathrm{Gli2}^{-/-}$skin and alleviate the Gli2 ${ }^{-/-}$follicular defects. We first established and characterized a stable line of K5-Gli2 transgenic mice (data not shown). Despite being expressed embryonically, K5-Gli2 transgenic mice do not exhibit any apparent defects in hair follicle development, but develop spontaneous tumors later in life, predominantly on their ears, similar to K5Gli2 transgenic founders (Grachtchouk et al. 2000). The K5-Gli2 transgene was then introduced into the Gli2 mutant background. Because K5-Gli2;Gli2 $2^{-/-}$mice die at birth, intercrosses between $\mathrm{K5}-\mathrm{Gli2} \mathrm{Gli2}^{+/-}$and $\mathrm{Gli2}^{+/-}$ mice were performed to generate E14.5 and E18.5 K5Gli2;Gli2-/- mice.

Consistent with the notion that Gli2 mediates Shh responses in the epithelium, the K5-Gli2 transgene restores epithelial expression of Gli1 and Ptc1 in E14.5 K5-Gli2;Gli2 ${ }^{-/-}$skin (Fig. 4N-P; data not shown). At E18.5, whereas most $\mathrm{Gli}^{-/-}$follicles are arrested at stage 2 of folliculogenesis (Fig. 4B,F), strikingly, K5Gli2; Gli2 $^{-/-}$follicles develop to more advanced stages (Fig. 4C,G), which are comparable to wild-type follicles (Fig. 4A,E). Similar to wild-type follicles (Fig. 4E,N,Q), K5-Gli2; Gli2 ${ }^{-/}$follicles exhibit correct compartmental- 


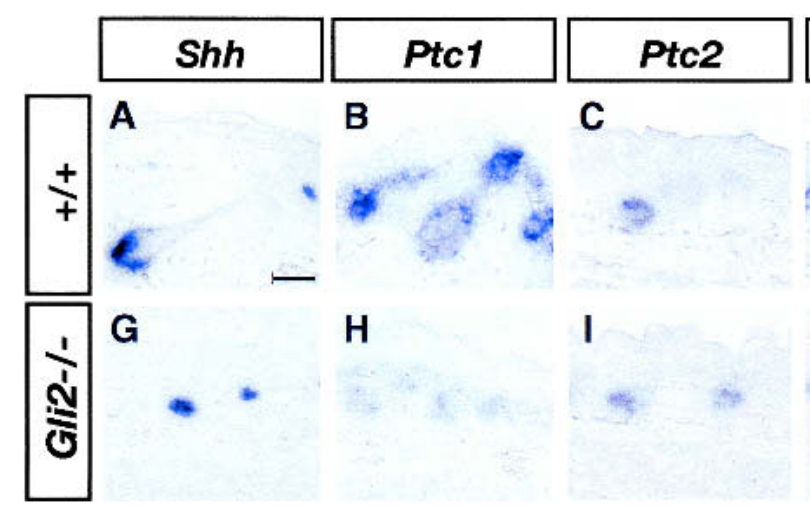

Figure 3. Shh responsiveness is reduced in Gli2-/- skin. Expression of Shh $(A, G)$, Ptc1 $(B, H)$, Ptc2 $(C, I)$, Gli1 $(D, J)$, Gli2 $(E, K)$, and $G l i 3(F, L)$ in E18.5 wild-type $(A-F)$ and $G l i 2^{-/-}(G-L)$ skin. The expression of the Shh-responsive genes, Ptc1 and Gli1, is dramatically reduced in Gli2 ${ }^{-/-}$mutants $(H, J)$. (M) Shh induces Gli1 and Ptc1 expression in dermal fibroblasts isolated from wild-type skin, as revealed by RT-PCR analysis. This Shh response is lost in Gli2 ${ }^{-/}$dermal fibroblasts.

ization (Fig. 4G) and high levels of Gli1 and Ptc1 expression (Fig. 4P,S). Within the follicular epithelium, keratinocyte proliferation in K5-Gli2;Gli2 ${ }^{-/-}$skin was restored to $95 \%$ of wild-type levels (Fig. 4T-V; p < 0.001), revealing a critical role of Gli2 in epithelial proliferation. Furthermore, xenografting experiments established that K5-Gli2;Gli2 $2^{-/}$grafts $(\mathrm{n}=4)$ develop further and produce functional hairs, with density and histology comparable to wild-type grafts (Fig. 4H-M). When Gli2 function is restored in $\mathrm{Gli}^{-/-}$epithelium, hair follicle development proceeds normally, establishing that the primary skin defect of $\mathrm{Gli2}^{-/-}$mutants lies in the epithelium. Importantly, despite the similarities between the follicular defects of $\mathrm{Gli2}^{-/-}$and $\mathrm{Shh}^{-/-}$mice, the K5-Gli2 transgene is incapable of rescuing the arrest of $\mathrm{Shh}^{-/}$ follicles (Fig. 4D), and K5-Gli2; $\mathrm{Shh}^{-1-}$ follicles do not express Shh target genes Ptc1 and Gli1 (data not shown; see Fig. 6, below). These results suggest that Gli2 requires a Shh-dependent event in order to function as an activator in the epithelium.

\section{Epithelial expression of Gli2 and $\Delta$ NGli2}

by a Cre-loxP based transgenic approach

To determine a possible Shh-dependence of Gli2 activator function, we examined whether a constitutive active form of Gli2 can bypass the requirement of Shh signaling for activation of Shh target genes, stimulation of epithelial proliferation, and hair follicle development. $\Delta$ NGli2, which lacks the $\mathrm{N}$-terminal repression domain, has been shown to be hyperactive in cultured cells and transgenic mouse embryos (Sasaki et al. 1999), suggesting that it may function as a constitutive activator form of Gli2.
We used a Cre-loxP-based conditional transgenic approach to achieve epithelial expression of Gli2 and $\Delta$ NGli2 in Gli2 and Shh mutant backgrounds.

We subcloned the Gli2 and $\triangle$ NGli2 cDNAs downstream of the $\beta$ geo cassette in the Z/AP double reporter construct (Fig. 5A; Lobe et al. 1999). Through ES cellmediated transgenesis and subsequent analysis, one $Z / A P$ Gli2 transgenic line and one $Z / A P-\Delta N G$ li2 transgenic line, which show similar level of transgene expression in the skin, were selected for the following studies. The triple polyadenylation sites (3xpA) located at the $3^{\prime}$ end of the Bgeo cassette terminate transcription and keep the Gli2 transgene and the associated human alkaline phosphatase reporter gene silent (Fig. 5B-D; data not shown). However, both the Gli2 transgene and the alkaline phosphatase reporter gene can be efficiently activated by Cre-mediated excision of the floxed $\beta$ geo cassette (Fig. 5E-G).

We first validated this transgenic approach by testing the effectiveness of the $Z / A P-G l i 2$ transgene in rescuing the follicular defects of $\mathrm{Gli}^{-/-}$skin. A $\mathrm{K} 5$-Cre transgene (Tarutani et al. 1997), which uses a K5 promoter similar to the K5-Gli2 transgene, was used to drive Cre-mediated activation of the $Z / A P-G l i 2$ transgene in the epithelium and outer root sheath. As shown by histology, the expression of Gli1 and Ptc1, and BrdU incorporation, K5-Cre;Z/AP-Gli2; Gli2 ${ }^{-/-}$mice $(\mathrm{n}=6)$ develop hair follicles (Fig. 6,O-Q) similar to those of wild-type mice (Fig. $6 \mathrm{~A}, \mathrm{D}-\mathrm{F})$. In the absence of Cre-mediated activation of the Gli2 transgene, Z/AP-Gli2;Gli2 ${ }^{-/-}$follicles (data not shown) are indistinguishable from $\mathrm{Gli}^{-/-}$follicles (Fig. 6B,G-I). These results confirm the notion that restoration of Gli2 function in the epithelium rescues the follicular defects of $\mathrm{Gli2}^{-/-}$skin. 

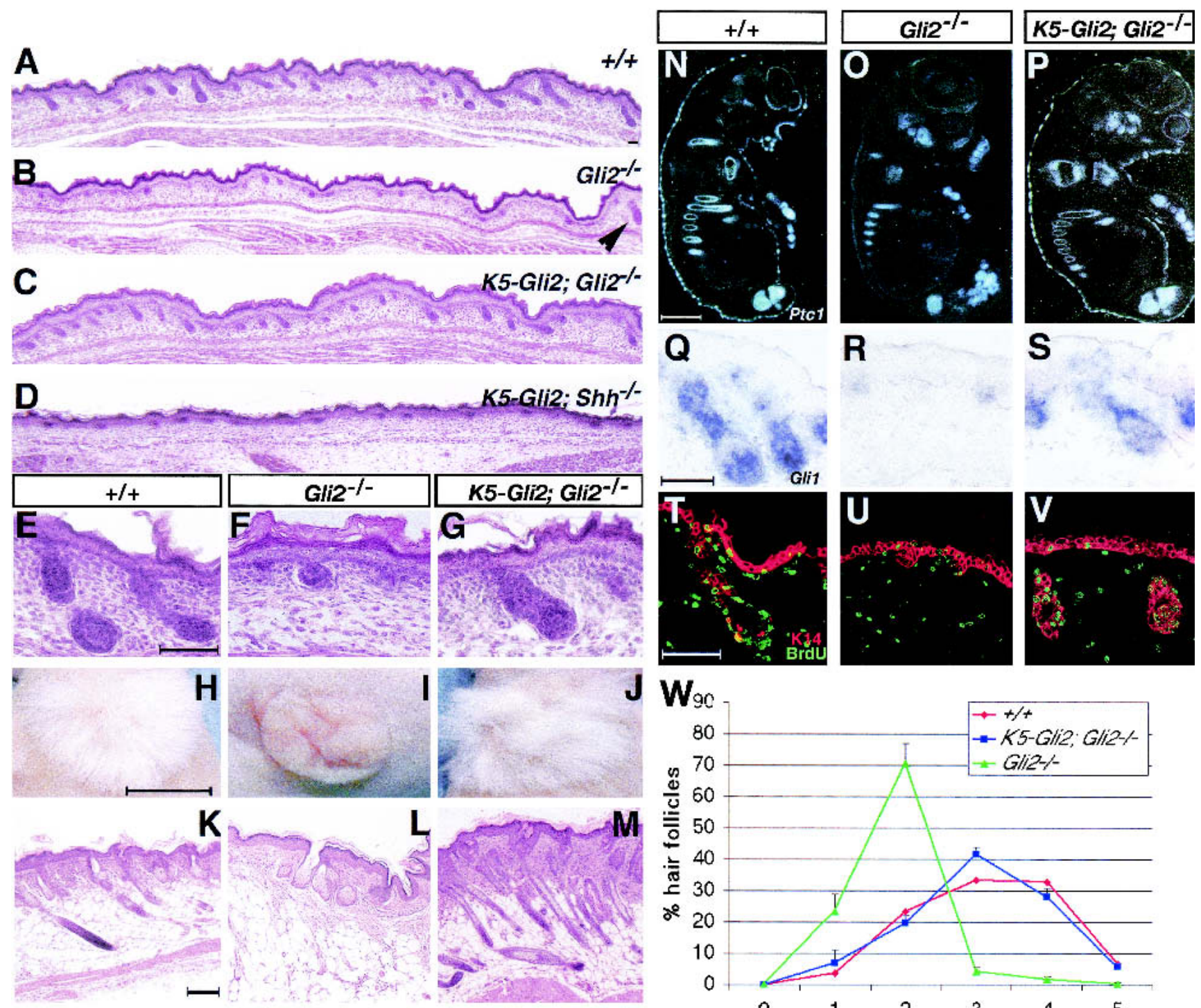

$\mathbf{J} \mathbf{W}_{90}$
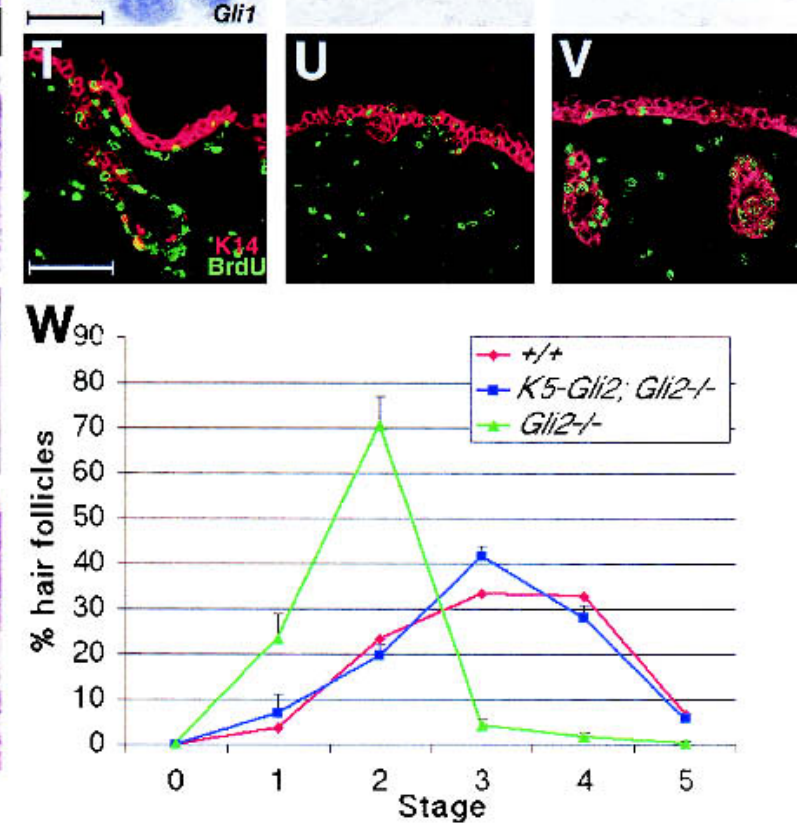

Figure 4. Epithelial Gli2 is sufficient to restore Shh responsiveness, stimulate proliferation, and rescue hair follicle development. $(A-G)$ Sections of E18.5 wild-type $(A, E), G l i 2^{-/-}(B, F), K 5-G l i 2 ; G l i 2^{-/-}(C, G)$, and K5-Gli2;Shh ${ }^{-/-}$dorsal skin at low $(A-D)$ and high $(E-G)$ magnification. $(B)$ Although most $G_{1 i 2}{ }^{--}$follicles are arrested at stages 1-2, some "escape" follicles (arrowhead) can develop further. $(H-M)$ Appearance $(H-J)$ and histology $(K-M)$ of wild-type $(H, K), G l i 2^{-/-}(I, L)$, and $K 5-G l i 2 ; G l i 2^{-/-}(J, M)$ skin xenografts after 50 $\mathrm{d}$ of incubation. (N-S) Dark-field illuminations of Ptc1 expression in E14.5 follicles in wild-type (N), Gli2 ${ }^{-/-}(O)$, and K5-Gli2;Gli2 ${ }^{-/-}$ $(P)$ skin. Gli1 expression by DIG in situ in wild-type $(Q), G_{1 i 2}{ }^{--}(R)$, and K5-Gli2;Gli2 ${ }^{-1-}(S)$ skin. (T-V) Staining of BrdU (green) and K14 (red) in E18.5 wild-type $(T)$, Gli2-/- $(U)$, and K5-Gli2;Gli2-/- $(V)$ skin. $(W)$ Differences in the progression of hair follicle development at E18.5: wild type (red), Gli2 ${ }^{-/}$(green), and K5-Gli2;Gli2 ${ }^{-/-}$(blue). Bars: $E-G, K-M, 50 \mu \mathrm{m} ; H-I, 2.5 \mathrm{~cm}$.

Similar to $\mathrm{K} 5-\mathrm{Cre}$;Z/AP-Gli2; Gli2-/- mice, $\mathrm{K} 5-\mathrm{Cre} ; \mathrm{Z} /$ $A P-\Delta N G l i 2 ; G l i 2^{-/-}$mice $(\mathrm{n}=6)$ also develop advanced stage hair follicles (Fig. 6U), exhibit a high level of Gli1 and Ptc1 expression (Fig. 6W, X), and show increased BrdU incorporation (Fig. 6Y), indicating that the $Z / A P-$ $\Delta$ NGli2 transgene is capable of rescuing the follicular defects of $\mathrm{Gli}^{-/-}$skin. However, some minor morphological differences are noted with the rescued follicles, which appear thickened along the shaft and base, and penetrate the dermis less deeply than do wild-type or K5-Cre;Z/AP-Gli2;Gli2 ${ }^{--}$follicles. Interestingly, Gli1 and Ptc1 are ectopically expressed in the interfollicular epithelium of $\mathrm{K} 5-\mathrm{Cre} ; \mathrm{Z} / \mathrm{AP}-\Delta \mathrm{NGli2} \mathrm{Gli2}^{-/-}$mice (Fig. $6 \mathrm{~W}, \mathrm{X})$, suggesting that $\Delta \mathrm{NGli2}$ is acting in a Shh-independent manner.

\section{$\Delta$ NGli2 activates Shh target gene expression and promotes epithelial cell proliferation in the absence of Shh}

To test whether $\Delta$ NGli2 is a Shh-independent activator of Shh target genes, we generated $\mathrm{K} 5-\mathrm{Cre} ; \mathrm{Z} / \mathrm{AP}-\mathrm{Gli2}$; 
A
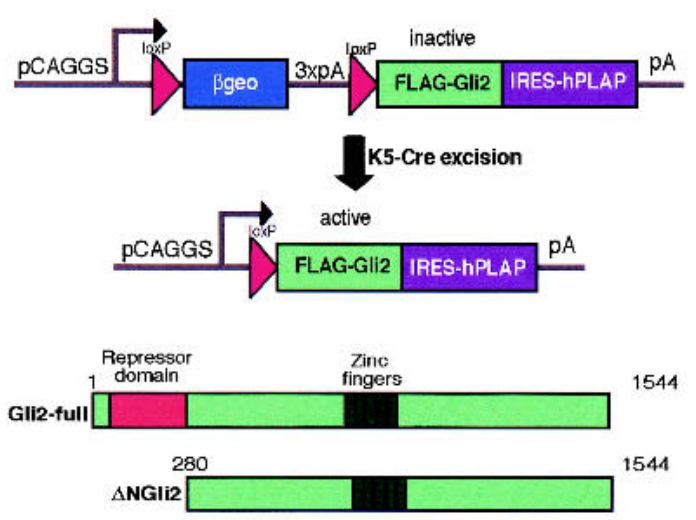

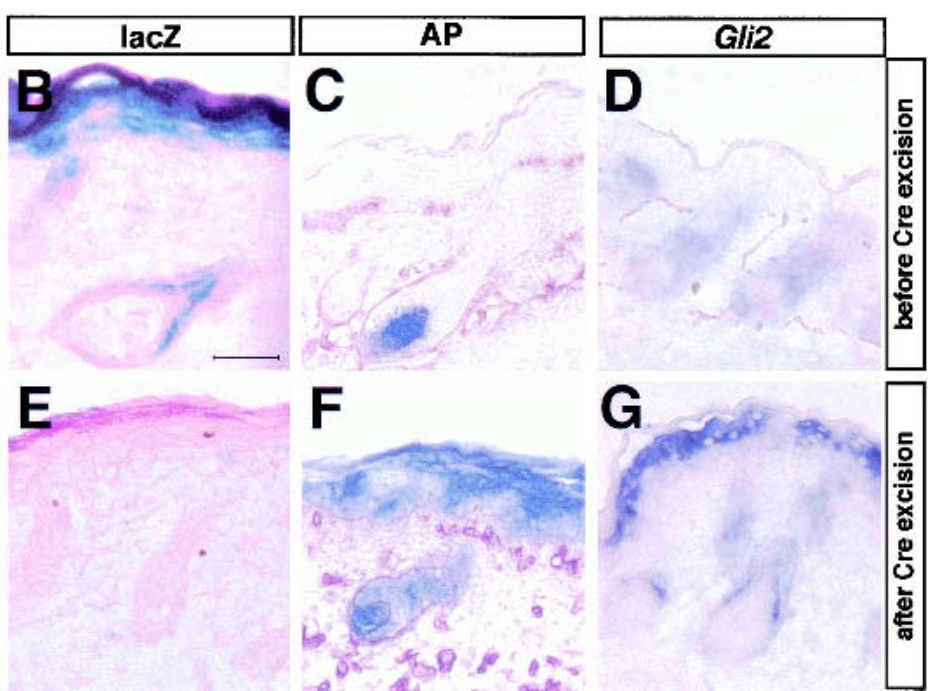

Figure 5. Inducible transgenic expression of Gli2 and $\Delta$ NGli2 in the developing epithelium. $(A)$ Schematic of the conditional Cre-loxP transgenic approach. The double reporter Z/AP construct (Lobe et al. 1999) contains a strong ubiquitous promoter that drives expression of reporter genes and Gli2. The first reporter, $\beta$ geo, which is followed by three polyadenylation signals, is flanked by lox $P$ sites (red arrowheads), which can be excised in the presence of Cre. The second reporter, human placental alkaline phosphatase (hPLAP), and Gli2 are expressed only after Cre excision. This strategy was used to target epidermal expression of full-length Gli2 and $\mathrm{N}$-terminally truncated Gli2, $\Delta$ NGli2 (Sasaki et al. 1999). Expression of the Z/AP- $\Delta$ NGli2 transgene in E18.5 skin in the absence $(B, C, D)$ and presence $(E, F, G)$ of $K 5-C r e .(B)$ In the absence of Cre, lacZ expression was found in all layers of interfollicular epithelium, infundibulum, and outer root sheath (ORS) of developing hair follicles. (C) hPLAP was not expressed in the absence of Cre; however, endogenous alkaline phosphatase activity is detected in dermal papillae. $(D)$ In situ hybridization revealed low levels of endogenous Gli2 expression in the epithelium and dermis. In the presence of Cre, K5-Cre;Z/AP- $\Delta$ NGli2 embryonic skin shows efficient excision of the $\beta$ geo cassette $(E)$, and induction of hPLAP $(F)$ and Gli2 transgene $(G)$ expression in the epidermis. Bar, $50 \mu \mathrm{m}$.

$\mathrm{Shh}^{-/-}$and $\mathrm{K5}-\mathrm{Cre} ; \mathrm{Z} / \mathrm{AP}-\Delta \mathrm{NGli2} ; \mathrm{Shh}^{-/-}$mice. Similar to K5-Gli2;Shh ${ }^{-1-}$ mice, K5-Cre;Z/AP-Gli2;Shh ${ }^{-1-}$ mice $(\mathrm{n}=7)$ show a skin phenotype indistinguishable from those of $\mathrm{Shh}^{-/-}$mice. All these mutant skin samples exhibit hair follicles arrested at the early hair peg stage (Fig. $6 \mathrm{C}, \mathrm{N})$ and show no epithelial or dermal expression of Shh target genes, Gli1 (Fig. 6J,R) and Ptc1 (Fig. 6K,S). Furthermore, there is little incorporation of BrdU (Fig. $6 \mathrm{~L}, \mathrm{~T})$, emphasizing the defect in proliferation, particularly in the epithelial compartment. Strikingly, the expression of Gli1 and Ptc1 (Fig. 6Z,AA) is restored in the developing follicles of K5-Cre; Z/AP- $\mathrm{N}$ NGli2; $\mathrm{Shh}^{-/-}$ mice $(\mathrm{n}=10)$ at levels comparable to those of wild-type follicles (Fig. 6D,E). Furthermore, ectopic expression of these Shh target genes is also detected suprabasally in the interfollicular epithelium of these mutants, as described earlier for K5-Cre; Z/AP- $\Delta$ NGli2; Gli2 $^{-/-}$mutants. These results provide genetic evidence indicating that $\triangle$ NGli2 is indeed a constitutively active form of Gli2, which can activate Gli1 and Ptc1 expression in a Shh-independent manner.

We next examined whether $\Delta$ NGli2 can promote epithelial cell proliferation in the absence of Shh. Increased follicular keratinocyte proliferation was observed in K5-Cre;Z/AP- $\Delta$ NGli2; $\mathrm{Shh}^{-/-}$skin, where the number of $\mathrm{BrdU}$ positive cells was 2.6-fold higher $(\mathrm{p}<0.005)$ as compared with $S h h^{-/-}$mutants (Fig. 6L,BB). A similar increase in PCNA staining was also observed in the follicles and interfollicular epithelium of $\mathrm{K} 5$
Cre;Z/AP- NGli2; $S h h^{-/-}$mutants (data not shown). These observations demonstrate the Shh-independent activity of $\Delta$ NGli2 as an effector of epithelial cell proliferation.

Although epithelial expression of Gli1 and Ptc1 as well as cell proliferation are elevated in $\mathrm{K} 5-\mathrm{Cre} ; \mathrm{Z} / \mathrm{AP}-$ $\Delta$ NGli2; $S_{S h}{ }^{-/-}$mice, the mutant skin does not exhibit a complete rescue of hair follicle development. When compared with $\mathrm{Shh}^{-1-}$ follicles (Fig. 6C), the majority of K5-Cre;Z/AP-SNGli2; Shh $^{-/-}$follicles are much larger and penetrate deeper into the dermis (Fig. 6V). Additionally, they exhibit more advanced follicular compartmentalization, including hair follicles with concave bases and stronger alkaline phosphatase activity of more rounded dermal condensates (see Supplemental Material). Although precise developmental staging of these follicles is difficult (Paus et al. 1999), expanded expression of K17, which marks the outer root sheath in later stages of hair follicle development, in $\mathrm{K} 5-\mathrm{Cre}$; Z/AP$\Delta N G l i 2 ; S h h^{-1-}$ mice further suggests that these follicles have bypassed some aspects of Shh follicle arrest (data not shown; McGowan and Coulombe 1998). Taken together, these results demonstrate that the activator function of Gli2 during folliculogenesis is Shh-dependent. However, the fact that $\Delta$ NGli2, a constitutive Gli2 activator, is not sufficient to program normal hair follicle development in the absence of Shh suggests that Shh signaling may play additional roles to the activation of Gli2 in the skin. 

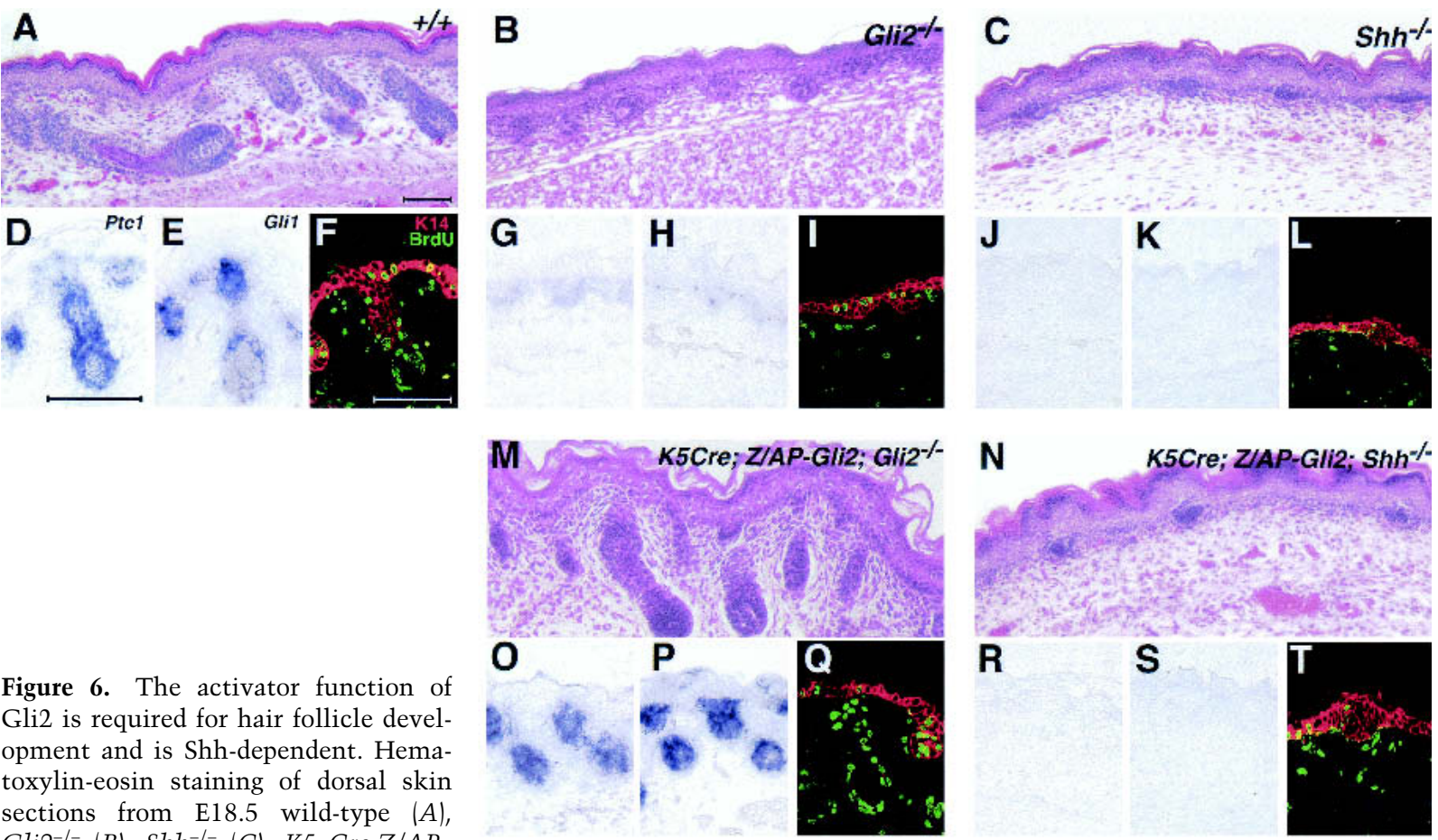

Figure 6. The activator function of Gli2 is required for hair follicle development and is Shh-dependent. Hematoxylin-eosin staining of dorsal skin sections from E18.5 wild-type $(A)$, Gli2 $^{-/-}(B), \mathrm{Shh}^{-/-}(C)$, K5-Cre; Z/APGli2;Gli2 $^{-1-}(M)$, K5-Cre;Z/AP-Gli2; $\mathrm{Shh}^{-1-}(N), \quad$ K5-Cre; Z/AP-A NGli2; Gli2 $^{-/-}(U)$, and K5-Cre;Z/AP- $\Delta$ NGli2; $\mathrm{Shh}^{-1-}(V)$ mice. In situ hybridization analysis of Shh target gene expression, Ptc1 $(D, G, J, O, R, W, Z)$ and Gli1 $(E, H$, $K, P, S, X, A A)$. Staining of BrdU (green) and $\mathrm{K} 14$ (red) in E18.5 wild-type $(F)$, $\mathrm{Gli2}^{-/-}(I), \mathrm{Shh}^{-/-}(L)$, K5-Cre;Z/APGli2;Gli2 $^{-/-}$(Q), K5-Cre;Z/AP-Gli2; $\mathrm{Shh}^{-1-} \quad(T), \quad$ K5-Cre;Z/AP- $D$ NGli2; Gli2 $^{-/-}(Y)$, and K5-Cre;Z/AP- $\Delta$ NGli2; $\mathrm{Shh}^{-/-}(B B)$ skin. Bars, $50 \mu \mathrm{m}$.
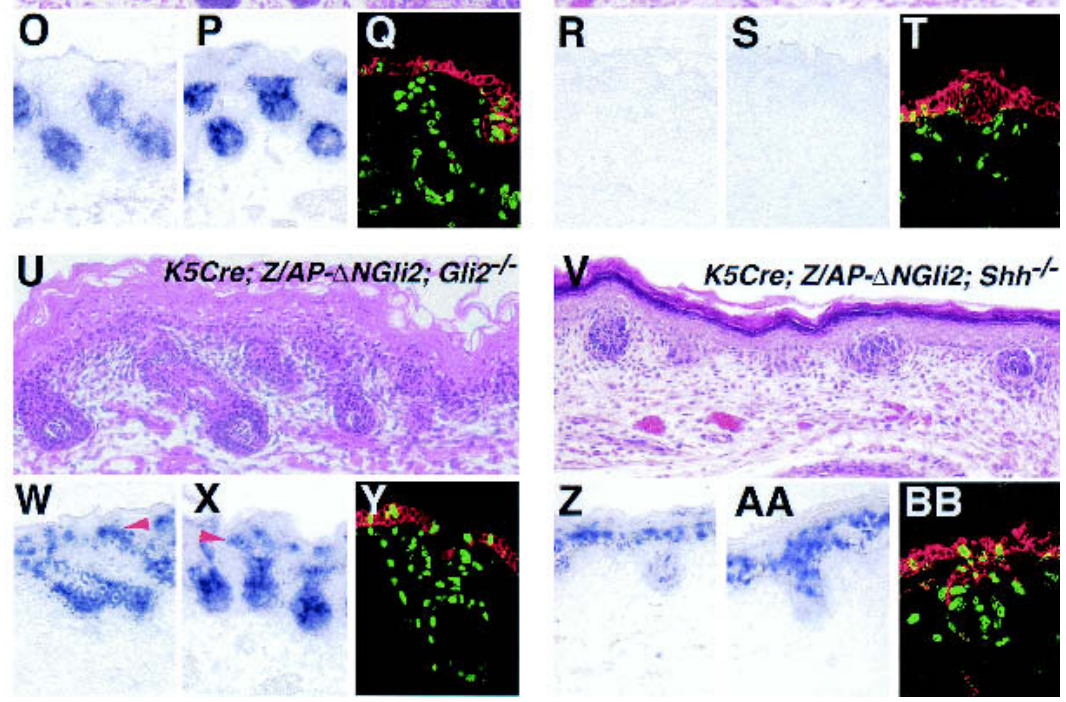

The Shh-dependent activator function of Gli2 regulates cyclin D1 and cyclin D2 transcription in developing hair follicles

Shh signaling promotes proliferation of primary human keratinocytes by opposing cell cycle arrest (Fan and Khavari 1999). Recent studies further demonstrate that $\mathrm{Hh}$ signaling can sustain cell cycle progression through upregulation of $\mathrm{G}_{1}$ cyclins, including cyclin D1, cyclin D2, and cyclin E (Kenney and Rowitch 2000; Long et al. 2001). To investigate the role of Gli2 in cell cycle control, we examined whether the Shh-dependent activation of Gli2 is involved in the regulation of D-type cyclins. In embryonic mouse skin, cyclin D1 is expressed at low levels in the basal layer of the interfollicular epithelium and throughout the developing follicles, cyclin D2 expression is prominent at the distal tip of developing follicles and appears to overlap with Shh expression, and cyclin D3 expression is detectable at very low levels in overlapping regions with cyclin D1 expression (Fig. 7A,B; data not shown). Whereas cyclin D1 expression is reduced in both $\mathrm{Gli2}^{-/-}$and $\mathrm{Shh}^{-/-}$skin (Fig. 7B,C), we find that there is little and no expression of cyclin D2 in $\mathrm{Gli2}^{-/-}$and $\mathrm{Shh}^{-/-}$follicles, respectively (Fig. 7E,F). Importantly, when Gli2 function is restored in the developing epithelium, cyclin D1 and cyclin D2 expression are restored to their endogenous patterns and levels in K5-Cre; Z/AP-Gli2; Gli22-l- rescued follicles (Fig. 7G,I). $\triangle$ NGli2, but not Gli2, can induce cyclin D1 and cyclin D2 expression in the absence of Shh $($ Fig. $7 \mathrm{H}, \mathrm{J}, \mathrm{L}, \mathrm{N})$, indicating that their transcription is regulated in part by the Shh-dependent activator function of Gli2. Interestingly, $\Delta$ NGli2 induces ectopic expression of cyclin D1, but not cyclin D2, in the suprabasal layers (Fig. $7 \mathrm{~K}-\mathrm{N}$ ). These results suggest that the Shh-dependent activator function of Gli2 promotes epithelial proliferation in embryonic skin through the transcriptional control of $\mathrm{D}$ type cyclins.

\section{Discussion}

Although it is known that Shh signaling is intimately involved in hair follicle development and skin tumori- 


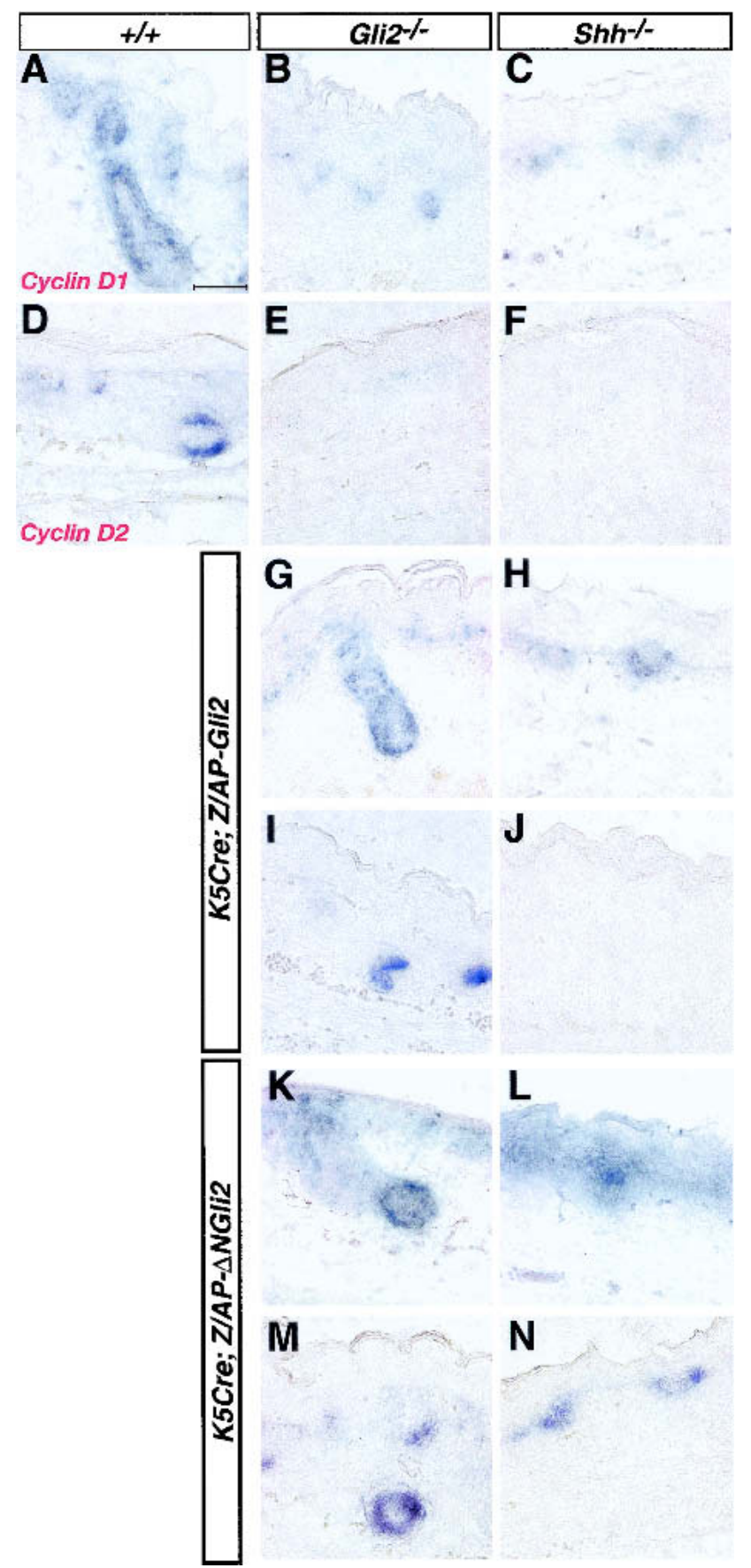

Figure 7. Gli2 activator regulates cyclin D1 and cyclin D2 expression in the developing epithelium. Expression of cyclin D1 $(A, B, C, G, H, K, L)$ and cyclin $D 2(D, E, F, I, J, M, N)$ in E18.5 wildtype $(A, D), G l i 2^{-/-}(B, E), S S^{-1-}(C, F), K 5-C r e ; Z / A P-G l i 2 ;$ Gli2 $^{-/-}(G, I)$, K5-Cre;Z/AP-Gli2;Shh ${ }^{-/}(H, J)$, K5-Cre;Z/AP$\Delta$ NGli2;Gli2 ${ }^{-/-}(K, M)$, and K5-Cre;Z/AP- $\Delta$ NGli2;Shh ${ }^{--}(L, N)$ mutants. Bar, $50 \mu \mathrm{m}$.

genesis, the details of the effectors and downstream events it coordinates in the skin remain elusive. Here, we show that Gli2 is the major activator mediating Shh responses during embryonic hair follicle development in mice. The $\mathrm{Gli2}^{-/-}$follicle defect phenocopies the ar- rested follicular development of $S h h^{-/-}$mutant mice, with reduced Shh-responsive gene expression and decreased cell proliferation. Through tissue-specific transgenic rescue experiments in mutant backgrounds, we have further dissected the role of Shh signaling in the developing skin and established that activation of Gli2 function in the epidermis by Shh signaling is essential for embryonic hair follicle development. (See Fig. 8 for a model.)

\section{Gli2 is a major mediator of Shh signaling in the skin}

Using a combination of genetic approaches, we illustrate that Gli2 is required for embryonic hair development. Gli2 $^{-/-}$skin shows defects in pelage follicle development strikingly similar to those observed in $S h^{-/-}$skin: arrest at early hair peg stage, reduced cell proliferation, and down-regulation of Shh target gene expression in both epithelial and dermal compartments. However, some escape hairs can still be found in Gli2 ${ }^{-/-}$skin. It is possible that Gli2 is not essential for the development of all pelage follicles, as there are several types of follicles in mice whose development initiate at different embryonic stages and appear to have different genetic requirements

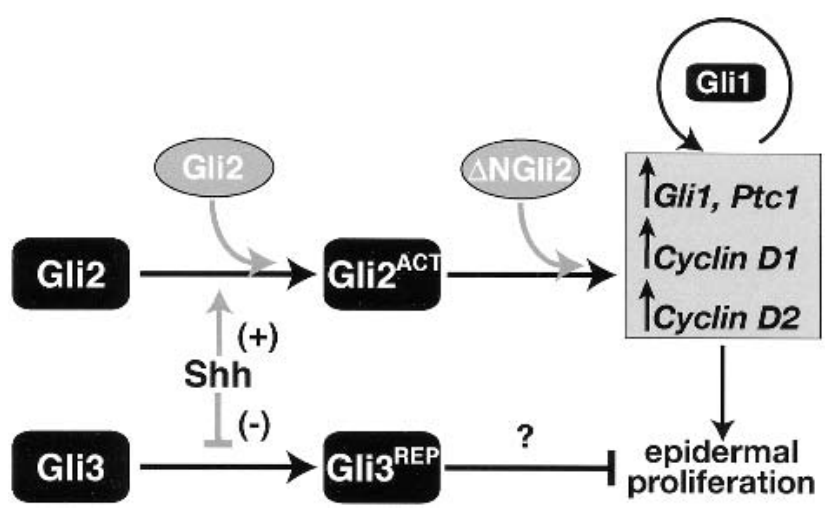

Figure 8. Model for Shh regulation of Gli activator and repressor forms in the embryonic hair follicle. In the developing hair follicles, Shh triggers a cascade of events leading to the formation of a potent transcriptional activator Gli2 $2^{\mathrm{ACT}}$ and inhibiting the formation of Gli repressors (e.g., Gli3). As the balance shifts to predominantly Gli activator forms, the expression of Shhresponsive genes, Ptc1 and Gli1, is induced. Gli1 itself is a potent transcriptional activator that can further potentiate the expression of Shh-responsive genes. Other Shh-responsive genes controlled by Gli activators include cell cycle regulators, such as cyclin D1 and cyclin D2, which are required for the proliferation of epidermal cells in the hair germ. In the absence of Shh, Gli activators will not be formed and Gli repressors will predominate. In our transgenic rescue experiments, we show that both Gli2 and $\Delta$ NGli2 can rescue hair follicle development in $\mathrm{Gli2}^{-/-}$skin, and that wild-type Gli2 requires Shh for its activation, whereas $\Delta$ NGli2 can bypass the requirement of Shh. Furthermore, our results suggest a role for Gli repressor in the developing skin, as $\Delta$ NGli2 cannot fully restore hair follicle development in $\mathrm{Shh}^{-/-}$skin, which is expected to possess a high level of Gli repressors (Gli3). (Black boxes) Endogenous Gli; (grey ovals) transgenic Gli. 
(for review, see Millar 2002). Alternately, a possible redundancy of Gli function in Shh signaling could exist in skin. This latter view is supported by the observation that escape hairs are not found in $\mathrm{Gli2}^{-/}$; $\mathrm{Gli3}^{+/-}$skin (data not shown). Together, these observations suggest that Gli2 is the major mediator of Shh signaling in the skin.

By transgenic rescue experiments, we show that the primary defect of $\mathrm{Gli2}^{-/-}$skin is in the epithelium. Although the development of dermal papillae is severely affected in Gli2 ${ }^{-/-}$skin and Gli2 ${ }^{-/-}$dermal fibroblasts fail to respond to Shh in vitro, restoration of Gli2 activity in the epithelium alone can fully rescue hair follicle development in $\mathrm{Gli2}^{-/-}$skin. Consistent with our results, it has been shown that Shh is insufficient to maintain the hair follicle-inducing characteristics of purified dermal papilla fibroblasts (Kishimoto et al. 2000). We propose that Gli2 can induce a secondary epithelial signal, which is subsequently required for maintenance and maturation of the dermal papilla.

\section{Gli2 activator function is Shh-dependent}

K5-Gli2 does not rescue $\mathrm{Shh}^{-/}$follicles, suggesting that Gli2 activator function is Shh-dependent. To test this, we used a conditional Cre-loxP transgenic approach to express a Shh-independent form of Gli2 in the epidermis (Lobe et al. 1999). $\Delta$ NGli2, which lacks the N-terminal repression domain, is a hyperactive form of Gli2 in activating Shh target gene expression both in vitro and in vivo (Sasaki et al. 1999). Importantly, $\Delta$ NGli2, but not Gli2, can induce Gli1 and Ptc1 expression in Shh ${ }^{-/-}$skin and induce ectopic expression of Gli1 and Ptc1 in regions normally lacking Shh response. These results indicate that $\Delta$ NGli2 functions as a constitutive activator of Gli1 and Ptc1 expression. In Drosophila, the activator function of $\mathrm{Ci}$ has been shown to be Hh dependent; an uncleavable form of $\mathrm{Ci}$, which is incapable of forming the repressor form, cannot induce the expression of target genes in the absence of Hh signaling (Méthot and Basler 1999). Although Gli2 can activate transcription in overexpression studies (see Sasaki et al. 1999), our data here clearly illustrate that the activator function of Gli2 in vivo is indeed Shh-dependent.

Although our results demonstrate the Shh-dependent activator function of Gli2, it remains unclear whether Gli2 also acts as a repressor. Several studies have suggested that, similar to $\mathrm{Ci}$ and Gli3, Gli2 can undergo proteolytic cleavage (Aza-Blanc et al. 2000; Wang et al. 2000a). Furthermore, in transgenic flies, Gli2 can function as a repressor and, interestingly, this repressor function is shown to be Shh-independent (Aza-Blanc et al. 2000). Although the physiological significance of the Gli2 repressor function has yet to be established, a study in which Gli1 was knocked into the Gli2 locus provides some insights into the functional similarity and difference between Gli1 and Gli2 in adult hair development (Bai and Joyner 2001). Whereas Glil can compensate for the lack of Gli2 function in Gli2 ${ }^{\text {Gli1KI }}$ mice during embryonic development, remarkably, adult Gli2 ${ }^{\text {Gli1KI mice }}$ develop skin defects, including alopecia and ulcers. These observations suggest that Glil could not perform an essential function of Gli2 during adult hair development. Although the molecular mechanisms await further investigation, it is plausible that Gli2 may possess a repressor function, which is lacking in Gli1, and this Gli2 repressor function plays a key regulatory role in the adult hair cycle.

$\Delta$ NGli2 can activate Gli1 and Ptc1 expression, and restore cell proliferation in $\mathrm{Shh}^{-/-}$epithelium, but the rescue of hair follicle development is not complete in K5-

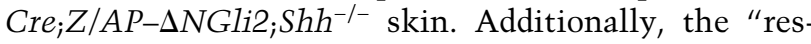
cued" follicles appear abnormal because they lack polarized downgrowth observed in wild-type skin (Fig. $6 \mathrm{~A}, \mathrm{U})$. Although we have restored the activator function of Gli2, K5-Cre;Z/AP- $\Delta$ NGli2; Shh ${ }^{-/-}$epithelium still contains high levels of Gli3 repressor function that might hinder hair follicle development. Indeed, an elevated level of Gli3 repressor has been shown to prevent ventral neural tube development and limb development in Shh ${ }^{-1-}$ mice (Litingtung and Chiang 2000; Litingtung et al. 2002). To address this possibility in skin, it will be important to study embryonic hair development in K5-

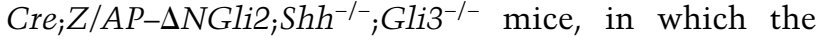
Gli2 activator function is provided and the Gli3 repressor function is removed in the epithelium.

\section{Gli2 activator function is required for epithelial proliferation}

In various developmental contexts, hedgehog signals have been linked to proliferative responses in target cells. Quiescent cells can be stimulated to enter the cell cycle in response to mitogenic signals, such as Shh, which induce the expression of D-type cyclins required to pass the $G_{1}$ restriction point. Shh has been shown to promote proliferation of cerebellar granule neuron precursors through up-regulation of cyclin D1, cyclin D2, and cyclin E mRNA and protein levels (Kenney and Rowitch 2000). Indian hedgehog signaling, which is required for chondrocyte proliferation, also regulates cyclin D1 expression in developing bone (Long et al. 2001). We show here that Shh signaling controls cyclin D1 and cyclin D2 expression in the developing follicle epithelium, suggesting a conserved role of Hh signaling in the regulation of D-type cyclins in diverse tissues. Shh-dependent activator function of Gli2 induces restricted expression of cyclin D2 and increased expression of cyclin D1, suggesting that the expression of cyclin D1 is controlled by both Shh-dependent and Shh-independent signals in embryonic skin. D-type cyclins are tightly regulated at both the transcriptional and posttranscriptional levels (Fantl et al. 1995; Sicinski et al. 1996). Similar to RNA expression, cyclin D1 and D2 proteins are down-regulated in Shh and Gli2 mutant skin and induced by Gli2 activator function (data not shown), with the exception that cyclin D1 protein is not stabilized in the suprabasal layers. Although no embryonic skin phenotype has been reported for either cyclin $D 1^{-/-}$or cyclin $D 2^{-/-}$mutant mice (Fantl et al. 1995; Sicinski et al. 1996), the overlap- 
ping expression and possibly redundant functions of the D-type cyclins in proliferating embryonic tissues could explain these observations. Indeed, cerebellar granule precursor neurons isolated from cyclin $D 1^{-/-}$or cyclin $D 2^{-/-}$mutants show wild-type proliferative responses on Shh stimulation in culture (Kenney and Rowitch 2000).

Importantly, our results illustrate that Gli2 activates cyclin D2 expression in a Shh-dependent manner, providing a molecular link between $\mathrm{Hh}$ signaling and cell cycle regulation. By microarray analysis, cyclin D2 has been identified as a putative transcriptional target of Gli1 in cultured epithelial cells (Yoon et al. 2002). Because both the human and mouse cyclin D2 promoters contain a consensus binding site for Gli proteins, it is highly plausible that cyclin $D 2$ represents a direct transcriptional target of Gli2 in the epithelium. A recent study has demonstrated that $\mathrm{Ci}$ can control cell growth and proliferation by directly regulating the expression of cyclin $D$ and cyclin E in Drosophila imaginal disc development (Duman-Scheel et al. 2002). Together, these observations suggest that up-regulation of D-type cyclins by Hh-dependent Gli activator function is evolutionarily conserved between mammals and flies. Hh signaling may additionally modulate cell cycle in a Gli-independent manner. It has been shown that Ptcl sequesters cyclin B1 in the cytoplasm and Shh can counteract this action, allowing nuclear localization of cyclin B1 (Barnes et al. 2001). More comprehensive analysis of cell cycle regulators is necessary to provide a better understanding of how Shh signaling regulates epidermal proliferation required for hair follicle development to proceed.

In this study we have shown that Shh signaling promotes the activation of Gli2 and, through transcriptional regulation of cyclin D1 and D2, Gli2 controls epithelial cell proliferation in embryonic skin. Similar Shh-dependent Gli2 activities may be involved in the regeneration of the secondary hair germs from hair follicle progenitors at the telogen-anagen transition during the adult hair cycle. By unraveling how Gli2 mediates these Shh-dependent mitogenic effects during development, we may gain important insight into the mechanisms by which abnormal activation of Shh signaling leads to uncontrolled expansion of tumor cells in skin cancers.

\section{Materials and methods}

\section{Mutant and transgenic Mice}

The generation and genotyping of mutant and transgenic mice were described previously: Gli2 and Gli3 (Mo et al. 1997); Shh (Chiang et al. 1996); K5-Gli2 (Grachtchouk et al. 2000); K5-Cre (Tarutani et al. 1997). Detailed procedures for the generation of the $Z / A P$ transgenic mice are available upon request. In brief, Gli2 and $\triangle$ NGli2 cDNAs (Sasaki et al. 1999) were cloned 3' of the loxP-flanked $\beta g e o-3 x p A$ cassette and $5^{\prime}$ of the IRES-hPLAP cassette in a pCAGG vector, and transgenic mice that carry a single copy of the Z/AP transgenes were generated through embryo stem cell-mediated transgenesis (Lobe et al. 1999).

Histology, alkaline phosphatase, and lacZ staining

Embryos were harvested and prepared for histology as described (Mo et al. 1997). Quantitative histomorphometry was carried out according to morphological and histological criteria (Paus et al. 1999) for 50 longitudinal hair follicles in sections of animals of each genotype $(n=8)$. Means and SEM were calculated from pooled data and differences were judged as significant if the $\mathrm{p}$ value was $<0.05$, as determined by an independent Student's $t$-test for unpaired samples. Staining for alkaline phosphatase and lac $Z$ activities were carried out as described (Lobe et al. 1999).

\section{In situ hybridization}

In situ hybridizations were carried out on paraffin sections as described (Mo et al. 1997; Motoyama et al. 1998a). Whole mount in situ hybridization was performed on embryos up to E15.5 (Motoyama et al. 1998a). The probes used in this study were as follows: Shh, Gli1, Gli2, Gli3 (Mo et al. 1997); Ptc1, Ptc2 (Motoyama et al. 1998b); K17 (P. Coulombe); cyclin D1 (V. Wallace); and cyclin D2 (Ambion).

\section{Immunohistochemistry, BrdU, and TUNEL labeling}

Immunohistochemistry was carried out as described (Chiang et al. 1999). Dilutions and other details concerning antibodies are available on request. For these studies, the following primary antibodies were used: laminin-5 (Chemicon); K14, K10, K6, K5, and Loricrin (Covance); AE13 (T.T. Sun); BrdU (Becton-Dickinson); Ki67 (DAKO); cyclin D1 (Oncogene); cyclin D1, cyclin D2, cyclin D3, PCNA, and p63 (Santa Cruz). Biotinylated secondary antibodies (Vector Labs) followed by either fluorescent streptavidin (Vector Labs) or the ABC Vectastain kit and VIP color substrate kit (Vector Labs) were used for visualization. For BrdU-labeling experiments, pregnant mice were injected with $0.1 \mathrm{mg} \mathrm{BrdU} / \mathrm{g}$ body weight and embryos were collected after 2 $\mathrm{h}$ and processed as described (Motoyama et al. 1998a). For apoptosis studies, an ApoAlert DNA fragmentation assay kit (Clontech) was used according to the manufacturer's directions. Sections were examined and photographed using either an Axioskop microscope or an Axiovert LSM 510 confocal microscope (Carl Zeiss).

\section{Skin grafts}

Full-thickness epidermal grafts from E18.5 embryos were transplanted onto dorsal excisions of the same size on adult nude mice. Grafts were allowed to develop for 4-6 wk prior to harvesting (St-Jacques et al. 1998).

\section{RT-PCR analysis of dermal cells}

Separation of E18.5 epithelium and dermis for the isolation of dermal cells was as described (Dlugosz et al. 1995). Briefly, dermal cells were cultured for $3 \mathrm{~d}$ in 6 -well plates, treated with recombinant Shh-N (gift from P. Beachy), and harvested $3 \mathrm{~d}$ later. RNA was isolated using Trizol and RT-PCR was performed as described (Chiang et al. 1999).

\section{Acknowledgments}

We thank P. Beachy, C. Chiang, P. Coulombe, C. Lobe, A. McMahon, H. Sasaki, M. Scott, T.T. Sun, and V. Wallace for reagents, and S.G. Kimmel, L. Wei, and J. Zhang for technical help. We appreciate insightful comments on the manuscript from B. Bruneau, S. Egan, T. Kunath, and J. Rossant. This work was supported by an NSERC Scholarship to P.M., a National Cancer Institute of Canada (NCIC) Scientist Award to C.c.H., 
funds from NCIC and Canadian Institute of Health Research to C.c.H., and NIH (R01 AR45973) to A.D.

The publication costs of this article were defrayed in part by payment of page charges. This article must therefore be hereby marked "advertisement" in accordance with 18 USC section 1734 solely to indicate this fact.

\section{References}

Aza-Blanc, P. and Kornberg, T. 1999. Ci: A complex transducer of the hedgehog signal. Trends Genet. 15: 458-462.

Aza-Blanc, P., Ramirez-Weber, F., Laget, M., Schwartz, C., and Kornberg, T. 1997. Proteolysis that is inhibited by hedgehog targets Cubitus interruptus protein to nucleus and converts it to a repressor. Cell 89: 1043-1053.

Aza-Blanc, P., Lin, H., Ruiz i Altaba, A., and Kornberg, T. 2000. Expression of the vertebrate Gli proteins in Drosophila reveals a distribution of activator and repressor activities. Development 12: 4293-4301.

Bai, C. and Joyner, A. 2001. Glil can rescue the in vivo function of Gli2. Development 128: 5161-5172.

Barnes, E., Kong, M., Ollendorff, V., and Donoghue, D. 2001. Patched1 interacts with cyclin $\mathrm{B} 1$ to regulate cell cycle progression. EMBO J. 20: 2214-2223.

Bitgood, M. and McMahon, A. 1995. Hedgehog and Bmp genes are coexpressed at many diverse sites of cell-cell interaction in the mouse embryo. Dev. Biol. 172: 126-138.

Chiang, C., Litingtung, Y., Lee, E., Young, K., Corden, J., Westphal, H., and Beachy, P. 1996. Cyclopia and defective axial patterning in mice lacking Sonic hedgehog gene function. Nature 383: 407-413.

Chiang, C., Swan, R., Grachtchouk, M., Bolinger, M., Litingtung, Y., Robertson, E., Cooper, M.K., Gaffield, W., Westphal, H., Beachy, P., et al. 1999. Essential role for Sonic hedgehog during hair follicle morphogenesis. Dev. Biol. 205: $1-9$.

Dahmane, N., Lee, J., Robins, P., Heller, P., and Ruiz i Altaba, A. 1997. Activation of the transcription factor Glil and the Sonic hedgehog signaling pathway in skin tumors. Nature 389: $876-881$.

Davidson, P. and Hardy, M. 1952. The development of mouse vibrissae in vivo and in vitro. J. Anat. Physiol. 86: 342-356.

Ding, Q., Motoyama, J., Gasca, S., Mo, R., Sasaki, H., Rossant, J., and Hui, C.-c. 1998. Diminished Sonic hedgehog signaling and lack of floor plate differentiation in Gli2 mutant mice. Development 125: 2533-2543.

Dlugosz, A., Glick, A., Tennenbaum, T., Weinberg, W., and Yuspa, S. 1995. Isolation and utilization of epidermal keratinocytes for oncogene research. Methods Enzymol. 254: $1-20$.

Duman-Scheel, M., Weng, L., Xin, S., and Du, W. 2002. Hedgehog regulates cell growth and proliferation by inducing Cyclin D and Cyclin E. Nature 417: 299-304.

Fan, H. and Khavari, P. 1999. Sonic hedgehog opposes epithelial cell cycle arrest. J. Cell. Biol. 147: 71-76.

Fantl, V., Stamp, G., Andrews, A., Rosewell, I., and Dickson, C. 1995. Mice lacking cyclin D1 are small and show defects in eye and mammary gland development. Genes \& Dev. 9: 2364-2372.

Fuchs, E. and Raghavan, S. 2002. Getting under the skin of epithelial morphogenesis. Nat. Rev. Genet. 3: 199-209.

Grachtchouk, M., Mo, R., Yu, S., Zhang, X., Sasaki, H., Hui, C.-c., and Dlugosz, A. 2000. Basal cell carcinoma in mice overexpressing Gli2 in skin. Nat. Genet. 24: 216-217.

Hardy, M. 1992. The secret life of the hair follicle. Trends Genet. 8: 55-61.
Hui, C.-c., Slusarski, D., Platt, K., Holmgren, R., and Joyner, A 1994. Expression of three mouse homologs of the Drosophila segment polarity gene Cubitus interruptus, Gli, Gli-2 and Gli-3, in ectoderm- and mesoderm-derived tissues suggests multiple roles during post-implantation development. Dev. Biol. 162: 402-413.

Ingham, P. and McMahon, A. 2001. Hedgehog signaling in animal development: Paradigms and principles. Genes \& Dev. 15: 3059-3087.

Iseki, S., Araga, A., Ohuci, H., Nohno, T., Yoshioka, H., Hayashi, F., and Noji, S. 1996. Sonic hedgehog is expressed in epithelial cells during development of whisker, hair and tooth. Biochem. Biophys. Res. Commun. 218: 688-693.

Karlsson, L., Bondjers, C., and Betsholtz, C. 1999. Roles for PDGF-A and sonic hedgehog in the development of mesenchymal components of the hair follicle. Development 126: 2611-2621.

Kenney, A. and Rowitch, D. 2000. Sonic hedgehog promotes $G_{1}$ cyclin expression and sustained cell cycle progression in mammalian neuronal precursors. Mol. Cell. Biol. 20: 9055 9067.

Kishimoto, J., Burgeson, R., and Morgan, B. 2000. Wnt signaling maintains hair-inducing activity of the dermal papilla. Genes \& Dev. 14: 1181-1185.

Litingtung, Y. and Chiang, C. 2000. Specification of ventral neuron types is mediated by an antagonistic interaction between Shh and Gli3. Nat. Neurosci. 3: 979-984.

Litingtung, Y., Dahn, R.D., Li, Y., Fallon, J.F., and Chiang, C. 2002. Shh and Gli3 are dispensable for limb skeleton formation but regulate digit number and identity. Nature 418: 979-983.

Lobe, C., Koop, K., Kreppner, W., Lomeli, H., Gertsenstein, M., and Nagy, A. 1999. Z/AP, a double reporter for Cre-mediated recombination. Dev. Biol. 208: 281-292.

Long, F., Zhang, X., Karp, S., Yang, Y., and McMahon, A. 2001. Genetic manipulation of hedgehog signaling in the endochondral skeleton reveals a direct role in the regulation of chondrocyte proliferation. Development 128: 5099-5108.

McGowan, K. and Coulombe, P. 1998. Onset of keratin 17 expression coincides with the definition of major epithelial lineages during skin development. J. Cell Biol. 143: 469-486.

Méthot, N. and Basler, K. 1999. Hedgehog controls limb development by regulating the activities of distinct transcriptional activator and repressor forms of Cubitus interruptus. Cell 96: 819-831.

Millar, S. 2002. Molecular mechanisms regulating hair follicle development. J. Invest. Dermatol. 118: 216-225.

Mo, R., Freer, A., Zinyk, D., Crackower, M., Michaud, J., Heng, H., Chik, K., Shi, X., Tsui, L., Cheng S., et al. 1997. Specific and redundant functions of Gli2 and Gli3 zinc finger genes in skeletal patterning and development. Development 124: $113-123$.

Motoyama, J., Liu, J., Mo, R., Ding, Q., Post, M., and Hui, C.-c. 1998a. Essential function of Gli2 and Gli3 in the formation of lung, trachea and oesophagus. Nat. Genet. 20: 54-57.

Motoyama, J., Takabatake, T., Takeshima, K., and Hui, C.-c. 1998b. Ptch2, a second mouse Patched gene is co-expressed with Sonic hedgehog. Nat. Genet. 18: 104-106.

Nilsson, M., Undén, A., Krause, D., Malmqwist, U., Raza, K., Zaphiropoulos, P., and Toftgård, R. 2000. Induction of basal cell carcinomas and trichoepitheliomas in mice overexpressing GLI-1. Proc. Nat1. Acad. Sci. 97: 3438-3443.

Oro, A., Higgins, K., Hu, Z., Bonifas, J., Epstein, E., and Scott, M. 1997. Basal cell carcinomas in mice overexpressing Sonic hedgehog. Science 276: 817-821.

Park, H., Bai, C., Platt, K., Matise, M., Beeghly, A., Hui, C.-c., 
Mill et al.

Nakashima, M., and Joyner, A. 2000. Mouse Gli1 mutants are viable but have defects in SHH signaling in combination with a Gli2 mutation. Development 127: 1593-1605.

Paus, R., Müller-Röver, S., van der Veen, C., Maurer, M., Eichmüller, S., Ling, G., Hofmann, U., Foitzik, K., Mecklenburg, L., and Handjiski, B. 1999. A comprehensive guide for the recognition and classification of distinct stages of hair follicle morphogenesis. J. Invest. Dermatol. 113: 523-532.

Sasaki, H., Nishizaki, Y., Hui, C.-c., Nakafuku, M., and Kondoh, H. 1999. Regulation of Gli2 and Gli3 activities by an amino terminal repression domain: Implication of Gli2 and Gli3 as primary mediators of Shh signaling. Development 126: 3915-3924.

Sato, N., Leopold, P., and Crystal, R. 1999. Induction of the hair growth phase in postnatal mice by localized transient expression of Sonic hedgehog. J. Clin. Invest. 104: 855-864.

Sicinski, P., Donaher, J., Geng, Y., Parker, S., Gardner, H., Park, M., Robker, R., Richards, J., McGinnis, L., Biggers, J., et al. 1996. Cyclin D2 is an FSH-responsive gene involved in gonadal cell proliferation and oncogenesis. Nature 384: 470474.

St.-Jacques, B., Dassule, H., Karavanova, I., Botchkarev, V., Li, J., Danielian, P., McMahon, J., Lewis, P., Paus, R., and McMahon, A. 1998. Sonic hedgehog signaling is essential for hair development. Curr. Biol. 8: 1058-1068.

Tarutani, M., Itami, S., Okabe, M., Ikawa, M., Tezuka, T., Yoshikawa, K., Kinoshita, T., and Takeda, J. 1997. Tissuespecific knockout of the mouse Pig-a gene reveals important roles for GPI-anchored proteins in skin development. Proc. Nat1. Acad. Sci. 94: 7400-7405.

Wang, B., Fallon, J., and Beachy, P. 2000a. Hedgehog-regulated processing of Gli3 produces an anterior/posterior repressor gradient in the developing vertebrate limb. Cell 100: 423434.

Wang, L., Liu, Z., Gambardella, L., Delacour, A., Shapiro, R., Yang, J., Sizing, I., Rayhorn, P., Garber, E., Benjamin, C., et al. 2000b. Conditional disruption of the Hedgehog signaling pathway defines its critical role in hair follicle development and regeneration. J. Invest. Dermatol. 114: 901-908.

Xie, J., Murone, M., Luoh, S., Ryan, A., Gu, Q., Zhang, C., Bonifas, J., Lam, C., Hynes, M., Goddard, A., et al. 1998. Activating Smoothened mutations in sporadic basal-cell carcinoma. Nature 391: 90-92.

Yoon, J., Kita, Y., Frank, D., Majewski, R., Konicek, B., Nobrega, M., Jacob, H., Walterhouse, D., and Iannaccone, P. 2002. Gene expression profiling leads to identification of GLI1binding elements in target genes and a role for multiple downstream pathways in GLI1-induced cell transformation. J. Biol. Chem. 277: 5548-5555. 


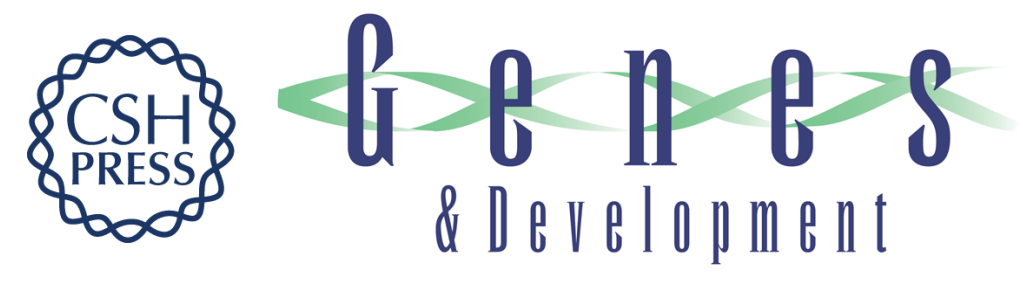

\section{Sonic hedgehog-dependent activation of Gli2 is essential for embryonic hair follicle development}

Pleasantine Mill, Rong Mo, Hong Fu, et al.

Genes Dev. 2003, 17:

Access the most recent version at doi:10.1101/gad.1038103

Supplemental
Material $\quad$ http://genesdev.cshlp.org/content/suppl/2003/08/11/17.2.282.DC2

References This article cites 47 articles, 16 of which can be accessed free at: http://genesdev.cshlp.org/content/17/2/282.full.html\#ref-list-1

License

Email Alerting

Receive free email alerts when new articles cite this article - sign up in the box at the top Service right corner of the article or click here.

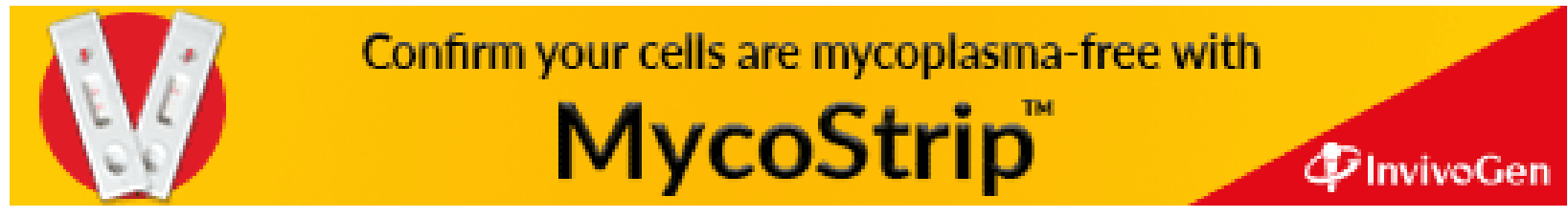

